\title{
Pertussis seroprevalence in adults, post-partum women and umbilical cord blood
}

\author{
Aurelia Fallo, M.D. ${ }^{a}$, Gabriela Manonelles, M.D. ${ }^{a}$, Daniela Hozbor, Biochemist ${ }^{b}$, \\ Claudia Lara, Biochemist ${ }^{c}$, Miguel Huespe, M.D. ${ }^{d}$, Silvina Mazzeo, M.D. ${ }^{d}$, \\ Oscar Canle, M.D. ${ }^{e}$, Marcelo Galas, Biochemist', and Eduardo López, M.D. ${ }^{a}$
}

\begin{abstract}
Pertussis is a vaccine-preventable disease that affects people of all ages. Young adults who have lost their immunity to pertussis are the major source of infection in infants. Given the steady increase of pertussis cases, new prevention strategies are required.

Objective. To assess pertussis seroprevalence in adult blood donors, post-partum women, and umbilical cords.

Metod. Measurement of total titers of antiBordetella spp. (Bordetella) antibodies using an enzyme-linked immunosorbent assay. Serum samples from 103 donors, 101 post-partum women and 100 umbilical cords were analyzed. Titers $\leq 80$ were considered of low impact against the disease. The assessment included transplacental transfer of antibodies and the umbilical cord/maternal ratio of antibody titers. Results. Donors mean age was: $28 \pm 6$ years old. Mediananti-Bordetellatiters:320;interquartilerange (IQR):160-320; 10\% had titers $\leq 80$.

Post-partum women mean age was: $26 \pm 6$ years old. Median anti-Bordetella titers:160 (IQR:80320 ), with titers significantly lower than in female donors $(p=0.00002)$. Titers $\leq 80$ were found in $30 \%$ of post-partum women.

Median anti-Bordetella titers in umbilical cords: 160 (IQR: 80-160). Titers $\leq 80$ were more frequently found in umbilical cords than in mothers $(44 \%$ versus $30 \%, p=0.04$ ). Transplacental transfer was 0.83 . Umbilical cord titers were equal to maternal titers in $54 \%$ of cases, lower in $37 \%$, and higher only in $8 \%$.

Conclusion. Titers of anti-Bordetella antibodies in post-partum women were significantly lower than in female blood donors. Titers $\leq 80$ were found in $30 \%$ of post-partum women and $44 \%$ of umbilical cords. These data may account for the high rates of pertussis in young infants who have not yet completed their vaccination schedule. Key words: pertussis, prevalence, prevention and control.
\end{abstract}

a. Department of Infectious Disease. Hospital de Niños Ricardo Gutiérrez, Buenos Aires.

b. National Reference Pertussis Laboratory. Universidad de La Plata, La Plata, Provinceof Buenos Aires.

c. National Reference Pertussis Laboratory- Clinical Bacteriology" Dr. Carlos G. Malbrán," Buenos Aires.

d. Department of Obstetrics and Gynecology. Hospital Santojanni, Buenos Aires.

e. Division of

Hemotherapy, Hospital de Niños Ricardo Gutiérrez, Buenos Aires.

E-mail Address: Aurelia Fallo, M.D.: afallo@intramed.net

Conflict of Interest: None.

Received: 9-16-2013 Accepted: 1-27-2014 http:/ /dx.doi.org/10.5546/aap.2014.eng.315

\section{INTRODUCTION}

Pertussis, also known as whooping cough, is an acute bacterial disease of the respiratory system caused by Bordetella pertussis (Bp). This disease mainly affects infants and toddlers. It is easily transmitted person-to-person, with an intra-family transmission rate of $75-90 \%$, while school transmission is $50-80 \%$, and can be endemic, with epidemic outbreaks every three to five years. ${ }^{1}$

The mass use of whole-cell vaccines reduced pertussis incidence by more than $95 \%$ in the 1980 s, compared to the 1940s-1950s pre-vaccine era.

However, over the past years, a steady increase of cases has been observed, even in countries with high vaccination coverages. ${ }^{2}$ The World Health Organizationestimates that 16 million cases of pertussis occur every year worldwide, with more than 194000 deaths, which account for 13\% of vaccine-preventable deaths. ${ }^{3}$

This is also the situation in Argentina, where a steady increase of cases has been observed since 2002 to date, with the highest morbidity and mortality rates observed among infants and toddlers. Ninety-two percent of hospitalized children are younger than one year old. In 2011, 70 infants died, 91\% were younger than four months old. ${ }^{4}$

Over the past two decades, a change has been recorded in the epidemiological profile of pertussis, with cases also detected among adolescents and adults. ${ }^{2}$ Although in these populations the disease is not so severe, it does have an epidemiological impact because adolescents and adults act as the main source of infection in infants. ${ }^{3-7}$

Different causes may epidemiological contribute to the present situation: greater surveillance, new diagnostic methods, a relatively low vaccine efficacy, short duration of vaccineinduced immunity, and/or the adaptability of the causative agent to the immunity conferred by vaccines. ${ }^{3}$ 
Regardless of causes, the significant increase in the number of cases has prompted health systems of different countries to review and implement new strategies aimed at reducing the incidence of pertussis in the most vulnerable population: infants younger than one year old. The precise mechanism that provides protective immunity to pertussis is not entirely known, but there is evidence of the role of both tumor and cellular response. ${ }^{8}$ Protection in infants younger than two months old (before receiving their first vaccine dose) depends on the transfer of maternal antibodies. Studies have been conducted on the role of such anti-Bordetella antibodies on the prevention of mortality in this group. ${ }^{9}$

Having our own data on the seroprevalence of pertussis in our population of young adults and post-partum women and on transplacental transfer of antibodies would be important at the time of designing new strategies aimed at improving the condition of this disease.

\section{OBJECTIVES}

- To assess the seroprevalence of anti-Bordetella antibodies in a population of young adults, including post-partum women and their umbilical cords.

- To compare the titer of antibodies in postpartum women with that of umbilical cord blood of their newborns.

\section{POPULATION AND METHODS}

Prospective, observational and analytical study with blind analysis of samples. Study period: June 2009-June 2010.

\section{Study population}

Group A: voluntary blood donors aged 1745 years old, called "donors."

Group B: women in their immediate postpartum period, called "post-partum women."

Group C:serum from their respective newborns collected from their umbilical cord blood, called "umbilical cords."

\section{Inclusion criteria}

Group A: healthy adult blood donors seen at the Hemotherapy Center of HNRG.

Group B: women with uneventful pregnancies reaching 37-41 weeks of gestation and giving birth at Hospital Santojanni.

Group C: umbilical cord blood serum from their infants, who were born healthy, at term and with an adequate weight for their gestational age.

\section{Exclusion criteria}

Adults who received gamma globulin or blood products in the past month.

Women with complicated pregnancies or deliveries.Umbilical cord blood from preterm newborn infants, those born with fetal anomalies or perinatal conditions.

\section{Ethical aspects}

The informed consent was obtained from all subjects included in the study, before blood donation in the case of donors, and before delivery in the case of mothers and umbilical cords.

The study was evaluated and approved by the ethics committees of hospitals Ricardo Gutiérrez and Santojanni.

\section{Methods}

For Groups A and B, questionnaires were completed in relation to demographic, epidemiological (cohabitation with children or adolescents, employment modality), and clinical data (history of prolonged cough, contact with confirmed cases of pertussis, concomitant conditions during pregnancy) and history of vaccination against pertussis and/or having had pertussis.

Serum samples were collected and stored at $-20^{\circ} \mathrm{C}$, then they were blindly sent to the laboratory, where they were all simultaneously processed.

The sample size was estimated using the EPI5 software for a population or descriptive study with a non-grouped randomized sampling and a $99.99 \%$ confidence interval (CI). It was expected that $50 \%$ of the general population and $45 \%$ of pregnant women had antibody titers $\geq 160$. For the estimation of the voluntary blood donor sample, we considered a population of 4000000 inhabitants aged 15-49 years old, based on the National Statistics and Censuses Institute of Argentina, which resulted in a sample size of $\mathrm{N}=79$. For the estimation of the post-partum women sample, we considered a population of 700000 deliveries/year, which resulted in a sample size of $\mathrm{N}=79$.

Titers of anti-Bordetella antibodies were determined using the enzyme-linked immunosorbent assay (ELISA), directed against whole-cell sonicates of B. pertussis strain Tohama I, which determines the level of total antibodies. Some studies have shown that titers $\geq 160$ are related to a past Bordetella infection; therefore, titers $\leq 80$ are considered to account for no evidence of protection against the disease. ${ }^{10}$

Transplacental transfer of antibodies was 
estimated as the ratio of the antibody titer geometric mean for umbilical cords serum and maternal serum.

In the analyses, antibody titers were expressed using the median (of the reciprocal of dilution) and its corresponding interquartile range (IQR). The statistical analysis of antibody titer medians was conducted using the Wilcoxon test for nonparametric samples. The qualitative outcome measure analysis was done using the Chi-square test or Fisher's test. A value of $p<0.05$ was considered significant.

\section{RESULTS}

Three-hundred and four samples were included and processed in the study, 103 were from voluntary donors, 101 from post-partum women, and 100 from umbilical cords. All included adults (donors and post-partum women) had received their last whole-cell vaccine dose at 6 years old (none had received the acellular vaccine). Group A consisted of 103 donors; 63 males and 40 females. Their mean age was $28 \pm 6$ years old (range: $17-$ 44 years old), with no gender differences (males: $28 \pm 6$ years old; females: $28.5 \pm 6$ years old) . Median titer of anti-Bordetella antibodies was 320 (IQR: 160-320), and 11 (10\%) had antibody titers $\leq 80$. When analyzing antibody titers by sex or age, no statistically significant differences were observed.

Donors who did not cohabitate with children tended to have lower titers than those who lived with children (15\% versus $8.6 \%$ ), but this was not a significant difference. In addition, no significant differences were detected between these two groups in terms of history of prolonged cough.

Group B consisted of 101 women in their immediate post-partum period. Their mean age was $26 \pm 6$ years old (range: 17-44 years old). Median titer of anti-Bordetella antibodies was 160 (IQR: $80-320) ; 30$ (30\%) of post-partum women had anti-Bordetella antibody titers $\leq 80$.

The analysis of antibody titers of post-partum women by age, history of prolonged cough or cohabitation with children showed no significant differences.

When comparing antibody titers between female donors and post-partum women, the latter had significantly lower titers, with a median of 160 (IQR: 80-320) versus 320 (IQR: 160-320) in female donors $(p=0.00002)$. At the same time, 30/101 $(30 \%)$ of post-partum women and only $2 / 40(5 \%)$ of female donors had anti-Bordetella antibody titers $\leq 80$, a remarkably significant difference (OR: 8.1; 95\% CI: 1.8-35.4, $p=0.0015$ ) (Figure 1).

FIGURE 1. Comparison of pertussis antibody titers in female donors $(N=40)$ and post-partum women $(N=101)$

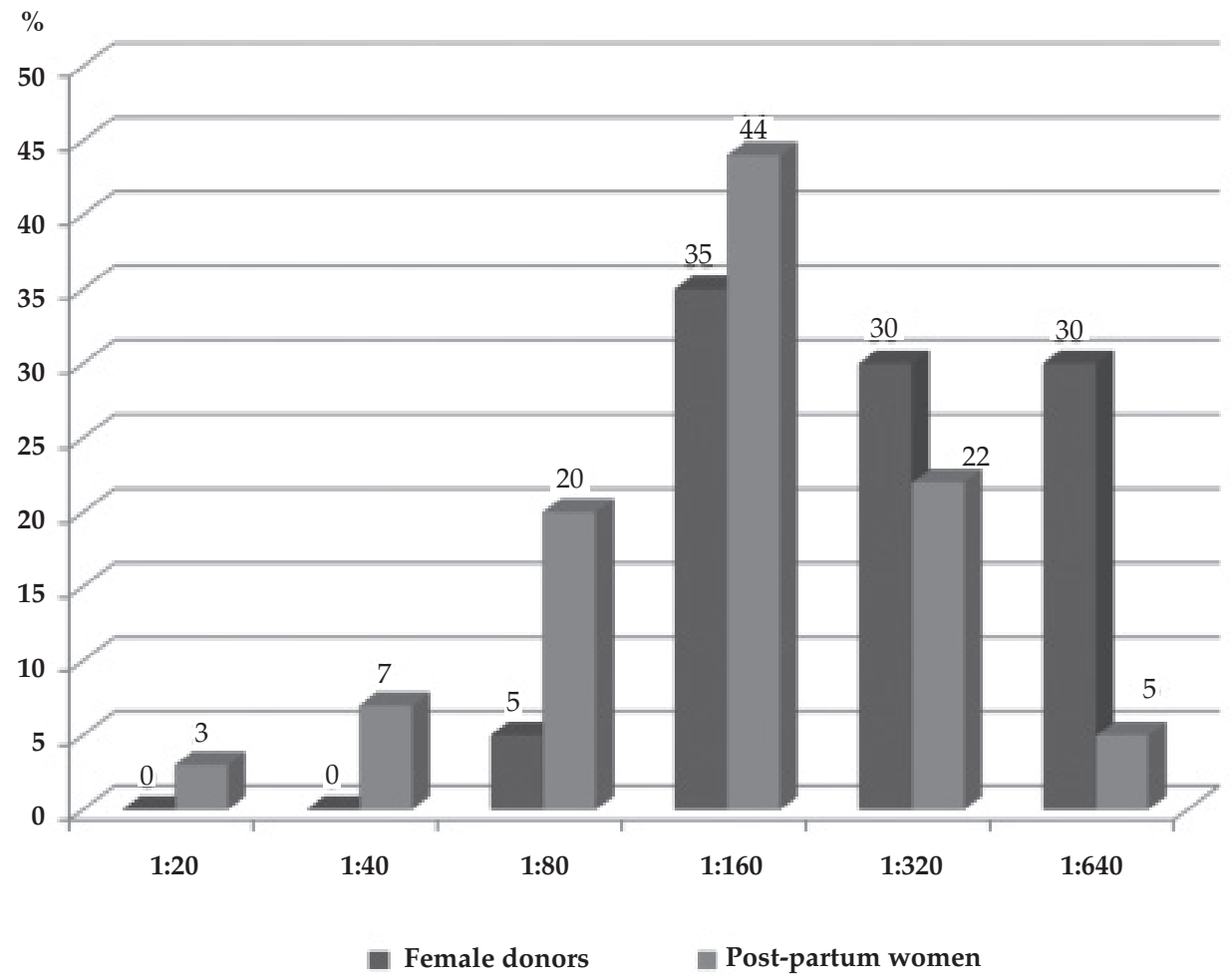


Figure 2 shows a comparison between titers of female donors and post-partum women by age, with the largest difference observed in young women.

The analysis of serum from 100 umbilical cordsshowed that the median titer of antiBordetella antibodies was 160 (IQR: 80-160), similar to maternal serum values, which showed a median of 160 (IQR: $80-320$ ). However, $44 \%$ of umbilical cords showed antibody titers $\leq 80$ versus $30 \%$ of post-partum women (OR 1.8; 95\% CI: 1.01$3.22, p=0.04)$.

Figure 3 shows antibody titers for each mothernewborn dyad, and demonstrates that umbilical cords titers were the same as in maternal serum in $54 \%$ of cases, lower in $37 \%$ of cases, and higher only in $8 \%$. The transplacental transfer ratio was 0.83 for the overall population, and in most age groups, cords had lower titers than maternal serum samples (Table 1).

\section{DISCUSSION}

As in other countries, the increased number of cases in Argentina has had a major impact on the morbidity and mortality of infants who have not completed their primary vaccination schedule. An increase in the number of cases in young adults who have lost their vaccine-induced immunity has also been reported. ${ }^{11-13}$
In a study conducted in our hospital during a pertussis outbreak (2007-2008) with 57 children hospitalized due to this condition, mean age of patients was 2.5 months old, with $80 \%$ younger than 6 months old and a $10 \%$ of deceased cases (all younger than 3 months old). The mean age of mothers was 24 years old, and $65 \%$ had a history of cough in the past month. ${ }^{14}$

The rate of pertussis cases in children aged 4-10 years old has remained stable. The fact that the highest rates occur in infants younger than six months old reflects that adults, especially young mothers, are the main reservoirs and sources of transmission, as demonstrated by several studies. ${ }^{15,16}$

This study provides the first data in Argentina regarding an important risk factor for pertussis in young infants, that is the serological "status" against pertussis in our young adult population (especially post-partum women) and at the time of birth. The methodology applied (total anti-Bordetella antibodies directed against whole-cell), the only one available in our country at the time of the study, allowed us to know the serological status of the assessed groups, with highly reproducible results and at an affordable cost.

At present, such methodology has been

FIGURE 2. Comparison of pertussis seroprevalence in female donors $(n=40)$ and post-partum women $(n=101)$ by age group

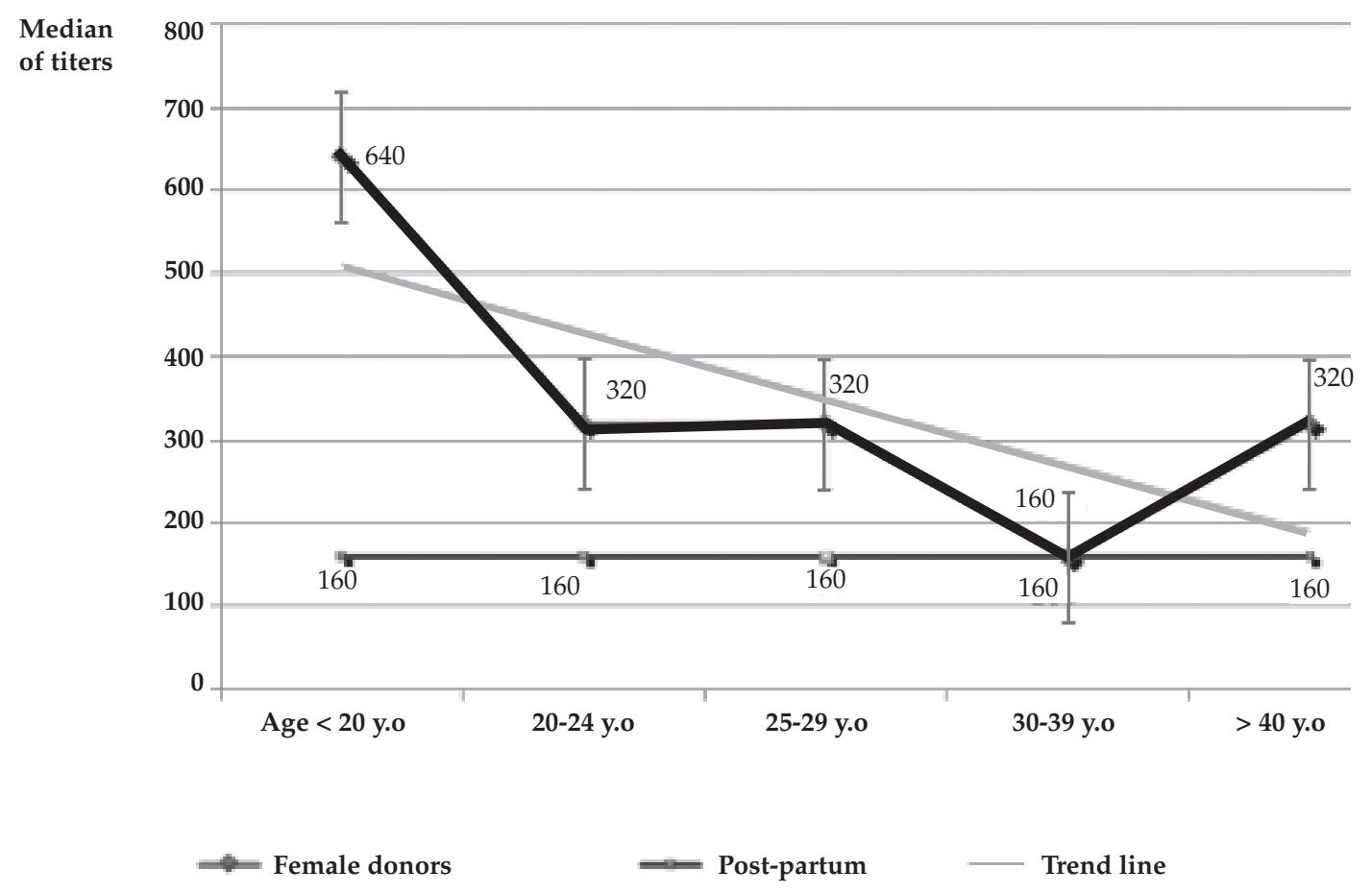


replaced by the measurement of pertussis antitoxin antibodies, which has a higher specificity, but is very expensive and still not available in our setting. Although no correlation has been established with protective immunity, there are sufficient data regarding the role of antibodies in infants younger than two months old. ${ }^{17}$

When analyzing the variation in titers in terms of participants' age, in the donor group it was observed that older adults had lower titers. However, in the post-partum women group, titers showed no variation in terms of age. Moreover, titers in this group were significantly lower than those of female donors.

In our cohort of mothers, 30\% had low titers $(\leq 80)$. A similar percentage was reported by Plans in Spain. ${ }^{18}$ Although it is difficult to compare the results of different studies because antibodies are measured with different methodologies, most studies report very low titers during pregnancy, probably as a result of their immunosuppressive status. ${ }^{17}$ This has also been reported in the response to other vaccines, including influenza. ${ }^{19,20}$

FIGURE 3. Comparison of anti-Bordetella antibody titers in each mother-cord dyad $(N=100)$

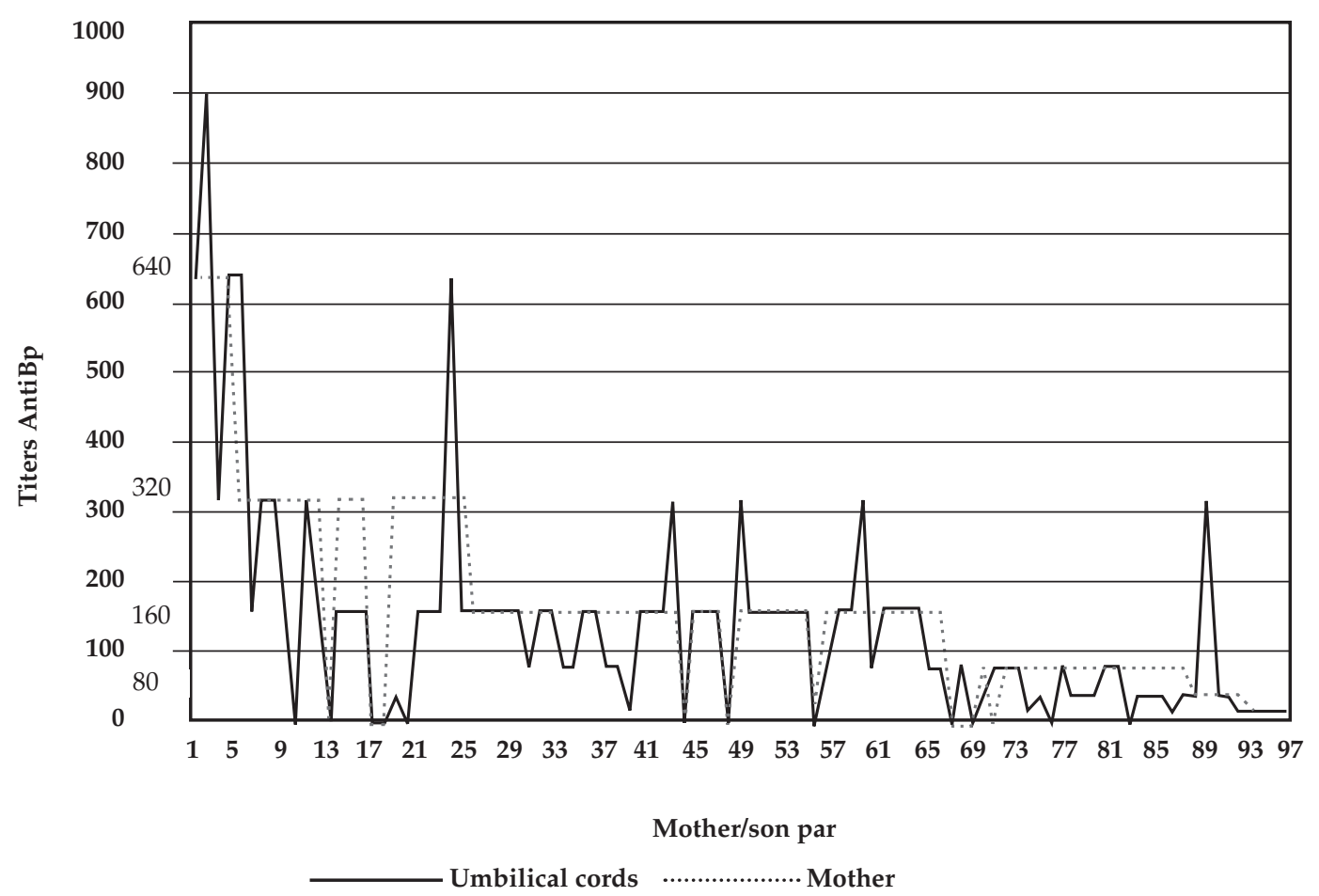

TABLE 1. Distribution of anti-Bordetella antibody titers in mothers, umbilical cords and mother-cord ratio, by maternal age group

\begin{tabular}{|c|c|c|c|}
\hline Age of mothers & $\begin{array}{c}\text { Geometric mean of } \\
\text { anti-Bordetella (range) } \\
\text { Umbilical cords }\end{array}$ & $\begin{array}{c}\text { Geometric mean of } \\
\text { anti-Bordetella (range) } \\
\text { Mothers }\end{array}$ & $\begin{array}{c}\text { Anti-Bordetella } \\
\text { Mother/ } \\
\text { umbilicalcords ratio }\end{array}$ \\
\hline$<20$ years old & $121(20-640)$ & $166(80-320)$ & 0.65 \\
\hline 20-24 years old & $155(20-2560)$ & $157(20-640)$ & 0.99 \\
\hline 25-29 years old & $113(40-640)$ & $128(40-640)$ & 0.88 \\
\hline 30-39 years old & $103(20-640)$ & $141(20-640)$ & 0.73 \\
\hline >40 years old & $67(20-160)$ & $95(20-160)$ & 0.71 \\
\hline All & $122(20-2560)$ & $146(20-640)$ & 0.83 \\
\hline
\end{tabular}

1. Anti-Bordetella antibodies.

2. Ratio between the geometric mean of umbilical cord antibody titers over the geometric mean of the maternal antibody titers. 
The analysis of antibody titers in umbilical cords showed that $44 \%$ had titers above 80 . Umbilical cord titers were the same as in the mothers in $54 \%$ of cases, lower in $37 \%$ and higher only in $8 \%$. The umbilical cord serum/maternal serum ratio was 0.83 . These results may suggest that placental transfer of antibodies is not as effective as proposed by other authors. ${ }^{19,21}$ Heinenger proposes an active transport mechanism in the transplacental transfer of antibodies, which probably starts around 17 weeks of gestation and reaches levels 1.5-2 times higher than in the mother when infants are born at term. ${ }^{19}$ Other studies reported similar maternal serum and umbilical cord serum levels. ${ }^{22}$ In a study conducted in 2004, Healy, et al. found that titers were higher in umbilical cords than in mothers (1.7), but a study from 2006 in Hispanic mothers reported similar antibody levels in both mothers and umbilical cords. The reason for such conflicting results among the studies is unknown, but Healy suggests that there are probably ethnic differences that could influence results. ${ }^{23-25}$

This study was done before Argentina implemented a vaccine dose in pregnant women, and supports the need to assess new strategies so as to improve the protection of the most vulnerable group, that is, infants in their first months of life.

Different studies have been conducted worldwide with the purpose of preventing pertussis in the first months of life. Different strategies have been studied, which are not necessarily mutually exclusive, but some still pose questions on their clinical effectiveness or implementation problems. Clearly, newly designed strategies should be based on a basic point, such as being able to maintain adequate vaccination coverages from usual schedules (complete schedule at $<18$ months old and boosters at 6 and 11 years old) because not achieving such critical objective would certainly render any other strategy unsuccessful.

Attempted strategies included:

- A booster dose in adolescents and young adults: its objective is to prolong the duration of vaccine-induced protection. The most important difficulty faced by this strategy is achieving adequate coverages. Its impact on young infants does not appear to be high. ${ }^{26}$

- Vaccination during the immediate post-partum period (cocooning): its objective is to protect susceptible mothers and indirectly protect their children, considering that the mother is the main source of transmission (50-75\%) during the first months of life. ${ }^{11,17,27}$ Ideally, any other susceptible person living with the infant should also be vaccinated so as to increase the potential success of this strategy. The most important difficulty in this case is to achieve an adequate coverage and the costs resulting from vaccinating all household members. ${ }^{25,28}$ Another item to be considered is that the peak serological response takes place fifteen days following the vaccination, leaving infants younger than two weeks old unprotected. ${ }^{29}$

- Neonatal vaccination: trials on neonatal vaccination with an acellular vaccine have generally demonstrated good results in terms of serological response as of fifteen days after immunization, except in Halasa's study. ${ }^{30-33}$ In Wood's study, a good response against pertussis was observed, but response titers with the hepatitis B and Haemophilus influenza vaccines were lower than in the control group. ${ }^{33}$ The fact that no monovalent vaccine has been authorized for use in the care environment should also be considered.

- Vaccination in pregnant women: this is potentially the only strategy that could provide protection since birth, and has been proposed after demonstrating that administering the vaccine to a pregnant woman after 20 weeks of gestation is safe and might convey protection. ${ }^{25,28-30}$ Antibodies transferred to the newborn infant could last approximately two months, when the usual vaccination schedule starts. ${ }^{34,35}$ The lack of data on clinical effectiveness and a possible interference with subsequent vaccinations have been reasons for concern. Heinenger's study reported a lower serological response to the usual vaccination schedule when wholecell vaccines are used. ${ }^{19}$

United States and Argentina have been the first countries to implement this latter strategy (2012). In the recommendations of both countries, it is emphasized that if the mother did not receive the vaccination during the pregnancy, it should be administered immediately in the post-partum period. ${ }^{28,35}$

We believe that, in the future, it would be important to widen these studies, verify results with a more specific methodology and, given that vaccination in pregnant women has been implemented, make comparisons between vaccinated and non-vaccinated populations, as well as study the progress of their infants so as to determine the impact of the strategy. 


\section{CONCLUSIONS}

The most relevant data obtained in this study are the low levels of antibodies detected in post-partum women and the even lower levels observed in umbilical cords, which might be consistent with the increased occurrence of pertussis in the first months of infants' life.

\section{REFERENCES}

1. American Academy of Pediatrics. Pertussis. En: Pickering LK, Baker CJ, Kimberlin DW, Long SS, eds. Red Book: 2009Report o! the Committee on In!ectious Diseases. 28th ed. Elk Grove Village, IL: American Academy of Pediatrics; 2009.Pages.504-19.

2. TanT,TrindadeE,SkowronskyD.Epidemiologyof pertussis. Pediatr Infect Dis J 2005;24:S10-S18.

3. Global Immunization Data, World Health Organization (WHO)/UNICEF, November2011.

4. Ministerio de Salud de la República Argentina, Boletín IntegradodeVigilanciaN.o 127,SE27, Vigilancia de Coqueluche, julio de 2012. Págs.15-19.

5. Bamberger E, Srugo I. What is new in pertussis? Eur J Pediatr 2008;167:133-9.

6. Bonmarin I, Lévy-Bruhl D, Baron S, Guiso N, et al. Pertussis surveillance in French hospitals: results from a 10 year period. Euro Surveill 2007;12:34-8.

7. Lasserre A, Laurent E, Turbelin C, Hanslik T, et al. Pertussis incidence among adolescents and adults surveyed in general practices in the Paris area, France, May 2008 to March 2009. Euro Surveill 2011;16(5). [Available at: http:/ /www.eurosurveillance.org/ ViewArticle.aspx/ ArticleId «19783].

8. Tran Minh NN, He Q, Edelman K, et al. Cell-mediated immune responses to antigens of Bordetella pertussis and protection against pertussis in school children. Pediatr Infect Dis J 1999;18(4):366-70.

9. Granstrom M, Olinder-Nielsen AM, Holmblad P, et al. Specificimmunoglobulin for treatment of whooping cough. Lancet 1991;338,1230-3.

10. Organización Panamericana de la Salud/OMS. Presente y futuro de las inmunizaciones. Washington DC: Organización Panamericana de la Salud; 1990. [Serie Paltex para ejecutores de programas de salud (ISBN 92 75710287); N.o22: 62].

11. Moore DM, Mathias RG. Patterns of susceptibility in an outbreak of Bordetella pertussis: evidence from a communitybased study. Can J Infect Dis 2002;13:305-10.

12. Wang Ch, Zhu QR. Seroprevalence of Bordetella pertussis Antibody in Children and Adolescents in China. Pediatr Infect Dis J 2011;30:593-6.

13. Greenberg D. Pertussis in adolescents increasing incidence brings attention to the need for booster immunization of adolescents. Pediatr InfectDis J 2005;24:721-8.

14 Manonelles G, Céccoli C, Fallo A, López E, et al. Impacto clínico-epidemiológico de la tos convulsa y análisis de costos en niños internados. Rev Hosp Niños B Aires 2009;51:3-9.

15. Bisgard KM, Pascual FB, Ehresmann KR, Miller CA, et al. Infant pertussis: who was the source? Pediatr Infect Dis J 2004;23:985-9.

16. Wendelboe AM, Njamkepo E, Bourillon A, Floret DD, et al. Transmission of Bordetella pertussis to young infants. Pediatr Infect Dis J 2007;26:293-9.

17. Healy CM, Munoz FM, Rench MA, Halasa NB, et al. Prevalence of pertussis antibodies in maternal delivery, cord, and infant serum. J Infect Dis 2004;190:335-40.
18. Plans P, Jansa J, Doshi N, Harrison TG, et al. Prevalence of pertussis antibodies in umbilical cord blood samples in Catalonia, Spain. Pediatr Infect Dis J 2008;11:1023-5.

19. Schlaudecker E, McNeal M, Dodd C, Ranz J, Steinhoff M. Pregnancy modifies the antibody response to trivalent influenza immunization. J Infect Dis 2012;206:1670-3.

20. Jamieson DJ, Theiler RN, Rasmussen SA. Emerging infections and pregnancy. Emerging Infectious Deseases 2006;12(11):1638-43.

21. Vanden BergJP,WesterbeekEA, Berbers GA, van Gageldonk PG, et al. Transplacental Transport of IgG Antibodies Specific for Pertussis, Diphtheria, Tetanus, Haemophilus influenzae Type b, and Neisseria meningitides serogroup c is lower in preterm compared with term infants. Pediatr Infect Dis J 2010;29:801-5.

22. Gonik B, Puder KS, Gonik N, Kruger Ml. Seroprevalence of Bordetella pertussis antibodies in mothers and their new born infants. Infect Dis Obstet Gynecol 2005;13:59-61.

23. Healy CM, Rench MA, Edwards KM, Baker CJ. Pertussis serostatus among neonates born to Hispanic women.Clin Infect Dis 2006;42(10):1439-42.

24. Castagnini LA, Healy CM, Rench MA, Wootton SH, et al. Impact of maternal postpartum tetanus and diphtheria toxoids and acellular pertussis immunization on infant pertussis infection. Clin Infect Dis 2012;54:78-84.

25. Healy CM, Rench MA, Castagnini LA, Baker CJ. Pertussis immunization in a high-risk post partum population. Vaccine 2009;27:5599-602.

26. American Academy of Pediatrics, Committee on Infectious Diseases. Preventionof pertussis among adolescents: recommendations for use of tetanus toxoid, reduced diphtheria toxoid, and acellular pertussis (Tdap) vaccine. Pediatrics 2006;117(3):965-77.

27. World Health Organization Pertussis vaccines: WHO position paper. Wkly Epidemiol Rec 2010;85:385-400.

28. Centers for Disease Control and Prevention. Updated recommendations for use of tetanustoxoid, reduced diphtheria toxoid and acellular pertussis vaccine (Tdap) in pregnant women and persons who have or anticipate having close contact with an infant aged $<12$ months-Advisory Committee on Immunization Practices (ACIP). 2011;60:1424-6.

29. Halperin BA, Morris A, Mackinnon-Cameron D, Mutch J, et al. Kinetics of the antibody response to tetanus-diphtheriaacellular pertussis vaccine in women of childbearing age and postpartum women. Clin Infect Dis 2011;53(9):885-92.

30. Belloni C, De Silvestri A, Tinelli C, Avanzini MA, et al. Immunogenicity of a three-component acellular pertussis vaccine administered at birth. Pediatrics 2003;111:1042-5.

31. Wood N, McIntyre P, Marshall H, Roberton D. Acellular pertussis vaccine at birth and one month induces antibody responses by two months ofage. Pediatr Infect Dis J 2010;29:209-15.

32. Halasa NB, O'Shea A, Shi JR, LaFleur BJ, et al. Poor immune responses to a birth dose of diphtheria, tetanus, and acellular pertussis vaccine. J Pediatr 2008;153:327-32.

33. Knuf M, Schmitt HJ, Jacquet JM, Collard A, et al. Booster vaccination after neonatal priming with acellular pertussis vaccine. J Pediatr 2010;156:675-8.

34. Leuridan E, Hens N, Peeters N, De Witte L, et al. Effect of a prepregnancy pertussis booster dose on maternal antibodytiters in young infants. Pediatr Infect Dis J 2011;30:608-10.

35. Ministerio de Salud de la Nación. PRONACEI. Manuales y lineamientos. Fundamentos de la vacunación de mujeres embarazadas con vacuna triple bacteriana acelular (Tdap). 2012. 